\title{
Targeted delivery of mesoporous silica nanoparticles loaded monastrol into cancer cells: an in vitro study
}

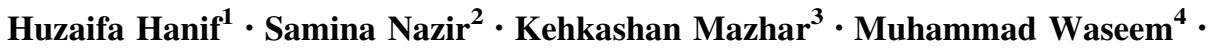 \\ Shazia Bano $^{2,5}$ - Umer Rashid ${ }^{6}$
}

Received: 18 July 2017/Accepted: 2 September 2017/Published online: 11 September 2017

(c) The Author(s) 2017. This article is an open access publication

\begin{abstract}
Monastrol is a simple low molecular weight dihydropyrimidine-based kinesin Eg5 inhibitor. Its low cellular activity and its non-drug-like properties have impeded its further development. In a previous report, we have reported various topological parameters to improve the pharmacokinetic properties of monastrol. The purpose of this study is to determine the loading and release feasibility of poorly water-soluble monastrol into the synthesized mesoporous silica nanoparticles (MSNs). The synthesis of MSNs was attained by the ammonia-catalysed hydrolysis and condensation of TEOS in ethanol using polysorbate- 80 as surfactant. These were characterized by BET surface area and pore size distribution analyses, SEM, XRD, UV and FTIR spectroscopy. The synthesized monastrol was successfully loaded on MSNPs and coated by hydrogels for successful controlled drug delivery. In vitro release studies are done by simple dialysis method.
\end{abstract}

Muhammad Waseem

waseem_atd@yahoo.com

$\triangle$ Umer Rashid

umerrashid@ciit.net.pk

1 Department of Chemistry, Hazara University, Mansehra, Pakistan

2 Nanoscience and Technology Department, National Centre for Physics (NCP) Islamabad, Islamabad, Pakistan

3 Institute of Biomedical and Genetic Engineering, Islamabad, Pakistan

4 Department of Physics, COMSATS Institute of Information Technology, Islamabad, Pakistan

5 Department of Physics, Islamia University, Bahawalpur, Pakistan

6 Department of Chemistry, COMSATS Institute of Information Technology, Abbottabad, Pakistan
Monastrol-loaded MSNPs were tested on human cervical epithelial malignant carcinoma (HeLa) cell lines for studying their anticancer activity. Our presented system described a reliable method for targeted delivery of monastrol into the cancer cells in vitro.

Keywords Mesoporous silica nanoparticles - Monastrol · KSP · Anticancer · Drug delivery

\section{Introduction}

The main objective in the field of nanomedicine is to acquire efficient delivery platforms for targeted delivery of therapeutic agents through proper nanomaterials. A serious interference in cancer treatment is due to the narrow range of operative biocompatible drug delivery systems. Mostly, low solubility in aqueous media is shown by the hydrophobic anticancer drugs like monastrol. Their inability to dissolve readily affects their activity in cancer treatment. Efficient drug delivery is very essential for enhancing diagnosis, reducing side effects and drug efficacy. Nanoparticles offer a capable approach towards delivering the therapeutic agents to the targeted organs and their use in cancer treatment (Nazir et al. 2014; Chen et al. 2016; Rosenholm et al. 2011; Wilczewska et al. 2012; Sahoo et al. 2007; Suri et al. 2007). Mesoporous silica nanoparticles are considered to be very promising candidate for drug delivery specifically for water-insoluble drug. Due to large surface areas and pores, these MSNPs act as reservoirs for storing hydrophobic drugs, whereas their shape and size can be altered according to the requirements (Zhang et al. 2012; Bharti et al. 2015). Several drugs with low water solubility have been explored to demonstrate this concept including ibuprofen, rifampin, etc. (Zhang et al. 
2010; Mohseni et al. 2015; Ganesh and Lee 2013). Based on non-toxicity and biocompatibility, silica nanoparticles are proposed as alternative carriers for drug delivery as these can be degraded into its simple silanol units and can be removed from the body (Fent et al. 2010; Yu et al. 2009; Liu et al. 2011). The porosity and shape of MSNPs accommodate the drug molecules. Therefore, for the effective drug loading and its delivery to the targeted sites, MSNPs are selective to capture bigger bioactive molecules in substantial amount (Jong and Borm 2008; Jaganathana and Godin 2012).

Dihydropyrimidines (DHPMs) are attractive scaffold for bioactivity because they can be easily altered and designed. Monastrol being a low molecular weight compound is revealed as a cell-permeable kinesin Eg5 inhibitor (Maliga et al. 2002). New class of anticancer agents directed by monastrol showed a significant progress in cancer therapy (Prokopcova et al. 2010). As monastrol is significant in targeting a motor protein, so it is helpful in examining the problems of spindle assembly in vitro and in vivo. Monastrol is fairly soluble in DMSO and ethanol but its solubility in water is poor. Further development of monastrol was halted due to its weak inhibitory activity as kinesin Eg5 inhibitor and non-drug-like properties (Schmidt and Bastians 2007).

In an effort to find potent urease inhibitors, we identified monastrol $\left(\mathrm{IC}_{50}=11.76 \mu \mathrm{M}\right)$ in structure-based virtual screening (SBVS) experiment (Rashid et al. 2013). In another report, we described a bioisosteric approach and reported various topological parameters to improve the pharmacokinetic properties of monastrol (Hassan et al. 2013). The main goal of the present work is to study the loading feasibility of monastrol into MSNPs, its release behaviour and targeted delivery into cancer cells. Here, we have prepared MSNPs which can enclose large amount of anticancer drug monastrol and tested its activity on HeLa cell lines (Fig. 1).

\section{Experimental}

\section{Chemicals used}

All the chemicals because of the highest quality were used as received. Anhydrous ethanol (99.9\%), $\mathrm{NH}_{3}$ (1\%), Polysorbate 80, Tetraethyl orthosilicate (TEOS), N-hydroxysuccinimide $98+\%$ (NHS), Folic acid, $\mathrm{N}$-(3dimethylaminopropyl)- $N$-ethylcarbodiimide hydrochloride (EDAC), Dimethyl Sulphoxide (DMSO), Chitosan, sodium carboxymethylcellulose (CMC), Acetic acid, TPP.

\section{Synthesis of monastrol}

Monastrol was synthesized by our reported procedure [17] using three component Biginelli reaction. 3-hydroxybenzaldehydes, ethyl acetoacetate and thiourea were reacted in a test tube under ultrasonic irradiation by using $\mathrm{SnCl}_{2}$ as catalyst and acetonitrile (ACN) as solvent (Scheme 1).

Yield $77 \%$. m.p. $185{ }^{\circ} \mathrm{C} .{ }^{1} \mathrm{H}$ NMR $(300 \mathrm{MHz}$, DMSO$\left.\mathrm{d}_{6}\right): \delta 1.09\left(t, 3 \mathrm{H}, J=7.2 \mathrm{~Hz}, \mathrm{OCH}_{2} \mathrm{CH}_{3}\right), 2.24(s, 3 \mathrm{H}$, $\left.\mathrm{CH}_{3}\right), 3.96\left(q, 2 \mathrm{H}, J=7.2 \mathrm{~Hz}, \mathrm{OCH}_{2}\right), 5.06(d, 1 \mathrm{H}$, $J=3.3 \mathrm{~Hz}, \mathrm{CH}), 6.60(m, 1 \mathrm{H}, \mathrm{Ar}-\mathrm{H}), 7.06(t, 1 \mathrm{H}, \mathrm{Ar}-\mathrm{H})$, 7.68 (br s, 1H, NH), 9.16 (br s,1H, NH) 9.37 (br s, 1H, OH). EIMS calculated for C14H16N2O3S $(\mathrm{M}+\bullet) 292.4$.

\section{Synthesis of mesoporous silica nanoparticles (MSNPs)}

The synthesis of hollow mesoporous silica nanoparticles was attained by the ammonia-catalysed hydrolysis and condensation of TEOS in ethanol using Polysorbate 80 as a surfactant. Typically, $1 \%$ solution of TEOS, $50 \mathrm{~mL}$ ethanol and $5 \mathrm{mM}$ tween 80 were mixed and stirred for half an hour and then $1 \%$ ammonia solution was added. The reaction mixture was kept overnight under stirring. The reaction mixture then turned turbid and the product thus formed was filtered after settling down the solution. MSNPs were obtained after calcination at $200{ }^{\circ} \mathrm{C}$ for $6 \mathrm{~h}$ and then at $400{ }^{\circ} \mathrm{C}$ for $4 \mathrm{~h}$.

\section{Loading of monastrol on silica nanoparticles}

Monastrol was dissolved in ethanol to which powdered MSNPs were added under constant magnetic stirring for $24 \mathrm{~h}$ at $37{ }^{\circ} \mathrm{C}$. The ratio of drug to nanoparticles was selected as $2: 1(\mathrm{w} / \mathrm{w})$. After $24 \mathrm{~h}$ of continuous stirring at $200 \mathrm{rpm}$, the particles were allowed to settle, centrifuge and wash with ethanol. Finally, the powdered monastrolloaded MSNPs were recovered after drying in open air at $37{ }^{\circ} \mathrm{C}$.

\section{Formation of hydrogel}

For creating a CMC and chitosan hydrogel, NPD conjugate solution (in ethanol) was mixed in CMC solution ( $0.01 \mathrm{~g}$ in $20 \mathrm{~mL}$ water) to which chitosan solution was added under constant magnetic stirring (200 rpm). The $\mathrm{pH}$ of the reaction mixture was maintained in the range 4-5 and then again CMC solution was added in drop-wise manner. For covalent linkages, TPP solution was added followed by constant agitation for further $30 \mathrm{~min}$. The appearance of turbidity thus confirms the formation of hydrogel. Finally, the solution was centrifuged to get gel encapsulated drugloaded silica particles. 
Fig. 1 Schematic representation of delivery of encapsulated monastrol into the cell

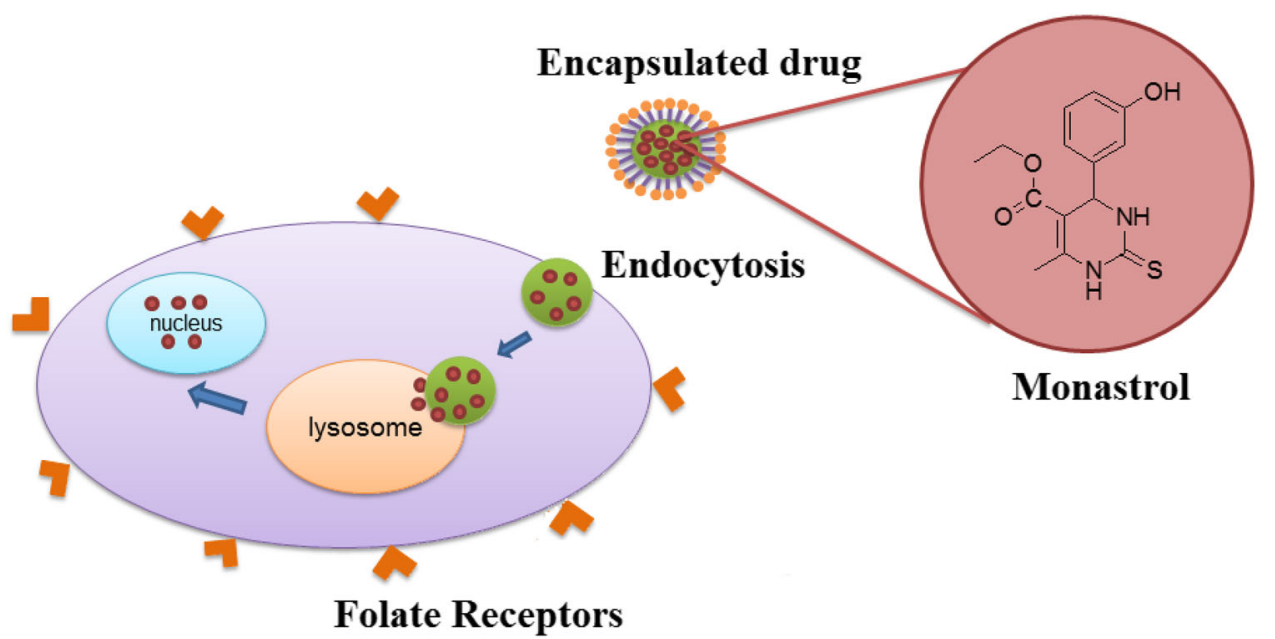

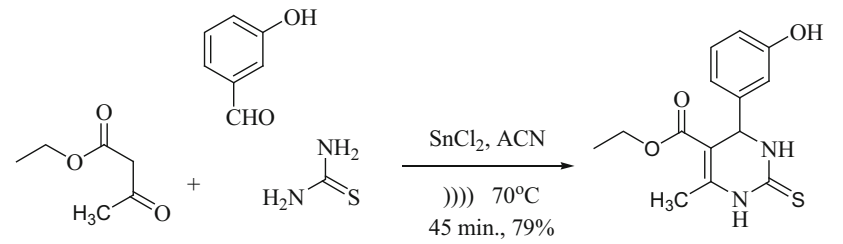

Scheme 1 Synthesis of monastrol

\section{Coating of folic acid}

For coating, $0.25 \mathrm{~g}$ folic acid was dissolved in $30 \mathrm{~mL}$ DMSO and sonicated for 15 min till a clear solution was obtained. Then, $0.225 \mathrm{~g}$ NHS and $0.125 \mathrm{~g}$ EDAC were added to folic acid solution and stirred for $2 \mathrm{~h}$. In this way, folic acid was activated. Afterward, $0.9 \mathrm{~g}$ gel was dissolved in $9 \mathrm{~mL}$ of ethanol to which $4 \mathrm{~mL}$ activated folic acid added and stirred for $4 \mathrm{~h}$. Finally, the gel products were centrifuged and air dried.

\section{MTT assay}

Human cervical epithelial malignant carcinoma (HeLa) cells were obtained from the American Type Culture Collection (Rockville, MD, USA) and grown in RPMI 1640 , supplemented with L-glutamine, $10 \%$ heat-inactivated Fetal Bovine Serum (FBS), $50 \mathrm{U} / \mathrm{mL}$ penicillin and $50 \mathrm{mg} / \mathrm{mL}$ streptomycin (Mediatech, Manassas, VA). The cells were maintained in a humidified incubator at $37{ }^{\circ} \mathrm{C}$ in an atmosphere of 5\% carbon dioxide. Monolayer cultures were seeded in 96-well plate $\left(10^{3}\right.$ cells/well) for $24 \mathrm{~h}$ before treatment with the nanoconstructs. Increasing concentrations of nanoconstructs were incubated with the cells for $24 \mathrm{~h}$ at $37^{\circ} \mathrm{C}$. The cell viabilities were measured using MTT assay.

MTT viability assay was prepared in a physiologically balanced solvent and cells were incubated with $100 \mu \mathrm{L}$ of
MTT solution for $3 \mathrm{~h}$ after removing the media. MTTcontaining media was replaced with $100 \mu \mathrm{L}$ of detergent and absorbance was measured on microplate reader (AMP PLATOS R-496) at the wavelength of $570 \mathrm{~nm}$. Active metabolic action in the viable cells converts MTT into a purple-coloured formazan product while the dead cells lose this ability, thus colour formation with an absorbance maximum near $570 \mathrm{~nm}$ serves as a suitable and useful marker of viable cells.

The percent viability was measured by subtracting noncellular background from samples relative to the nontreated cells (NTC) as negative control using formula:

$\left(\mathrm{OD}_{\text {treated }} / \mathrm{OD}_{\text {control }}\right) \times 100$,

where OD is the optical density at $570 \mathrm{~nm}$. Phase contrast images were obtained to observe cellular morphology.

\section{Results and discussion}

\section{Characterization of MSNPs}

The surface area and pore size distribution studies of MSNPs were performed by $\mathrm{N}_{2}$ adsorption method at $77 \mathrm{~K}$ by means of surface area analyser model Quantachrome NOVA 1200e. Prior to the analysis, silica sample was degassed at $373 \mathrm{~K}$ for $2 \mathrm{~h}$. The specific surface area and pore size distribution of silica were measured by Brunauer Emmett and Teller (BET) and Barrett, Joyner and Halenda (BJH) methods, respectively. Figure 2 represents the $\mathrm{N}_{2}$ adsorption-desorption isotherm of MSNPs, whereas the inset represents the pore size distribution. According to IUPAC classification, the adsorption/desorption isotherm represents type IV having $\mathrm{H} 1$ type of hysteresis loops which is the characteristic of mesoporous material. The BET surface area of MSNPs was found to be $328 \mathrm{~m}^{2} / \mathrm{g}$. The isotherm further shows that there was a steep 


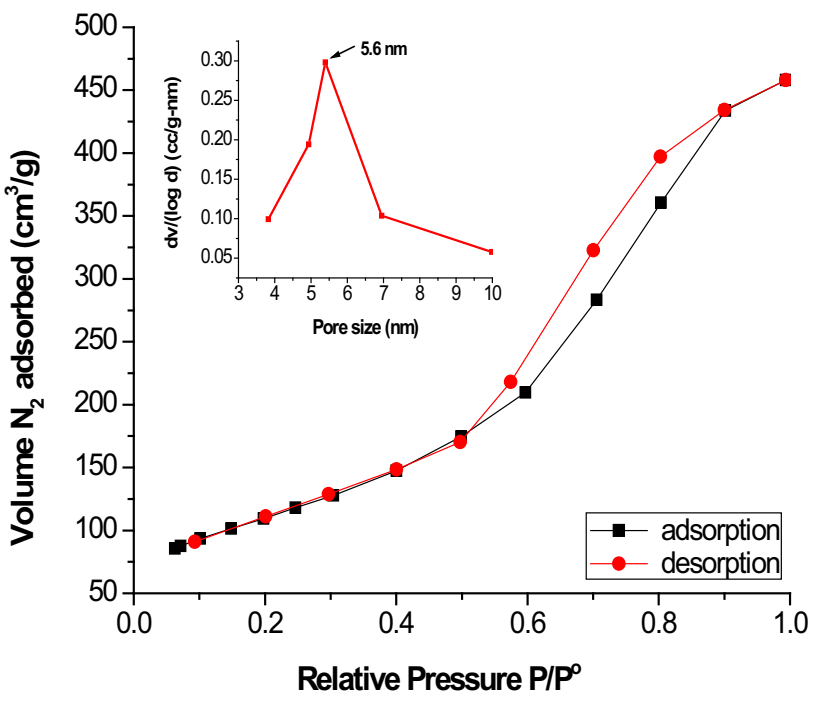

Fig. $2 \mathrm{~N}_{2}$ adsorption/desorption isotherm of MSNPs (Inset pore size distribution)

adsorption and desorption of $\mathrm{N}_{2}$ when $P / P^{\mathrm{o}}<0.05$ which points towards the presence of micropore in the matrix. However, at relatively higher pressure $\left(P / P^{\mathrm{o}}>0.06\right)$, there was a marked difference in the adsorption and desorption of $\mathrm{N}_{2}$ indicating the presence of larger pores filled at higher relative pressure. The pore size distribution (PSD) was calculated by plotting pore size vs. desorption cumulative volume and it shows that the maximum pore size of silica particles was about $5.6 \mathrm{~nm}$ which further confirms that the silica particles were of mesoporous in nature.

Surface morphology, particle size and the microscopic features of MSNPs were observed with SEM (JEOL JSM5910). The micrograph was depicted in Fig. 3, which shows that silica NPs are clearly visible (inset of the figure) and are nearly spherical in shape with the size distribution $45-60 \mathrm{~nm}$.

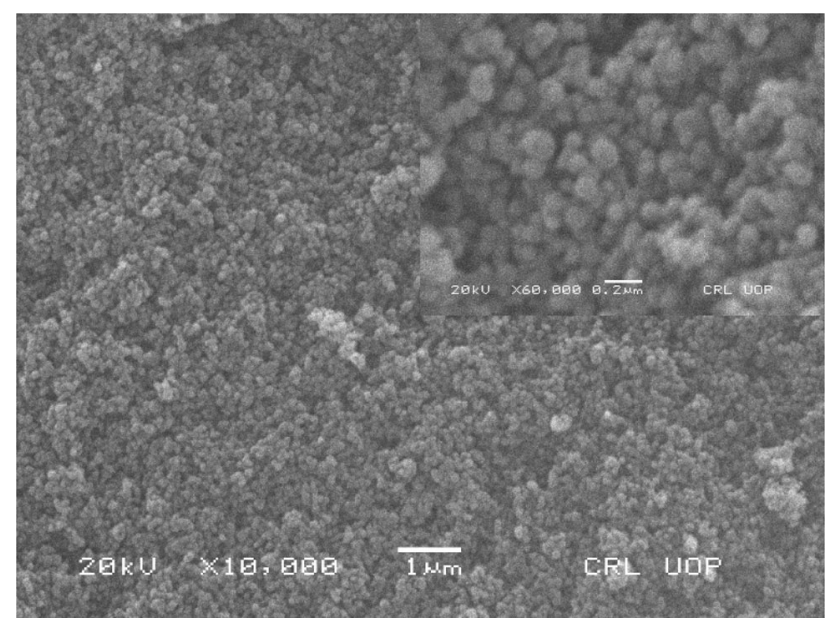

Fig. 3 SEM micrograph of mesoporous silica NPs

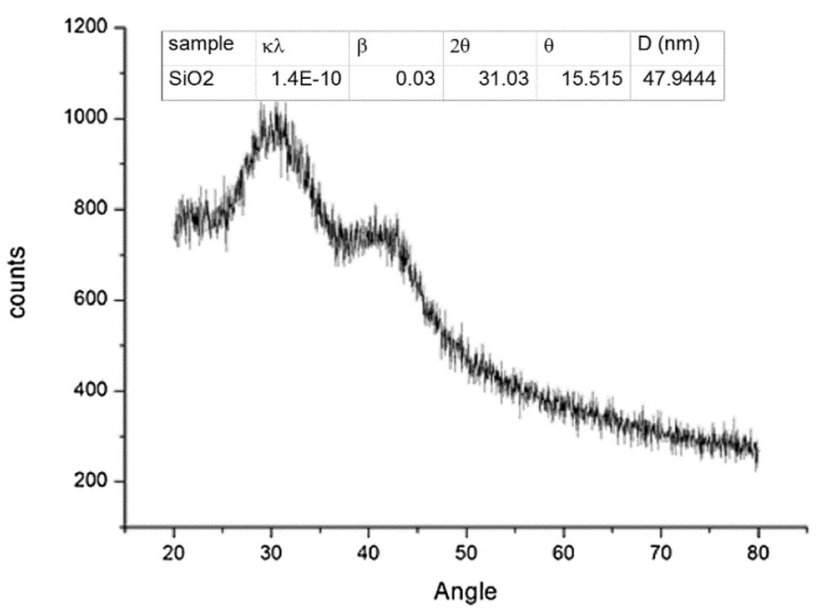

Fig. 4 X-rays diffractogram of mesoporous silica NPs

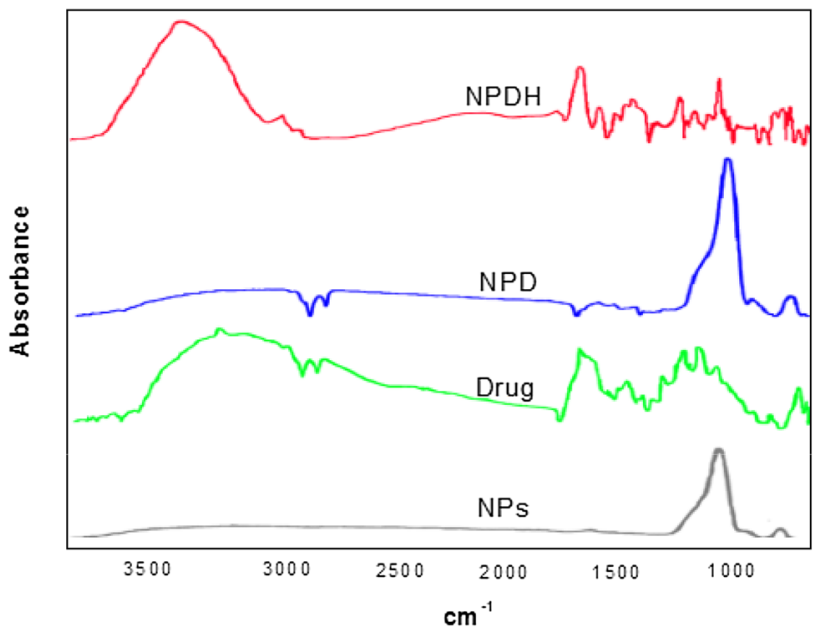

Fig. 5 FTIR spectra of mesoporous silica NPs before and after drug loading

In order to determine the nano-crystalline size of MSNPs, X-ray powder diffraction (XRD) measurements were recorded at room temperature in Bruker D8 ADVANCE diffractometer equipped with $\mathrm{Cu}-\mathrm{K} \alpha$ radiation $(\lambda=1.54056 \mathrm{~A})$. The average calculated crystallite size was $47.94 \mathrm{~nm}$; however, the particle size from the SEM image was found close to the diffraction data. The diffraction data have been shown in the inset of Fig. 4 .

FTIR spectroscopy was performed to trace the functional groups before and after the loading of drug molecules on mesoporous silica nanoparticles and the spectrum (Fig. 5). Before taking IR measurements, translucent sample discs were prepared by mixing powder sample with $\mathrm{KBr}$. The samples were then loaded in FTIR spectroscope (Bruker, Tensor 27) with a scan range $400-4000 \mathrm{~cm}^{-1}$ at a resolution of $4 \mathrm{~cm}^{-1}$. The samples of present interest were nanoparticle (NP), drug, nanoparticle-drug conjugate 
(NPD) and nanoparticle-drug-hydrogel (NPDH) as differentiated in the given spectra. From the FTIR spectra, it is clearly indicated that silica NPs showed a silicon absorption band position $1076.06 \mathrm{~cm}^{-1}$ which can be associated to $\mathrm{Si}-\mathrm{O}-\mathrm{Si}$ (Waseem et al. 2010). The band observed at $3162 \mathrm{~cm}^{-1}$ is due to $\mathrm{OH}$ stretching vibrations of water molecules. In the spectrum of drug, the absorption bands of various functional groups like amine, amide and aromatic ring are observed. After drug loading on mesoporous silica nanoparticles, the silicon absorption band positioned at $1076.06 \mathrm{~cm}^{-1}$ was detected along with the band associated to the functional groups present on drug molecules. The IR studies therefore, confirm the successful loading of drug molecules onto MSNPs. The formation of hydrogel on NPD conjugate was further confirmed by several bands obtained as similar of chitosan and CMC.

Figure 6 represents the optical absorption spectra taken in the range of $250-800 \mathrm{~nm}$ at room temperature and were measured by UV-VIS spectrophotometer model Perkin Elmer UV/VIS-Lambda 25. Contrarily to mesoporous amorphous silica, UV spectrum of drug monastrol gives a peak at $320 \mathrm{~nm}$ showing that monastrol absorbs light in this region. The successful loading of drug onto NPs (NPD) was confirmed after getting a sharp peak at $300 \mathrm{~nm}$. Afterward a formation of hydrogel on NPD was performed by coating of CMC and chitosan (NPDH). The Chitosan was found UV inactive while its presence around NPD display significant absorption; however, it was found less than NPD. Finally folic acid is loaded on hydrogel (NPDHF) for which the absorbance up to two was detected.

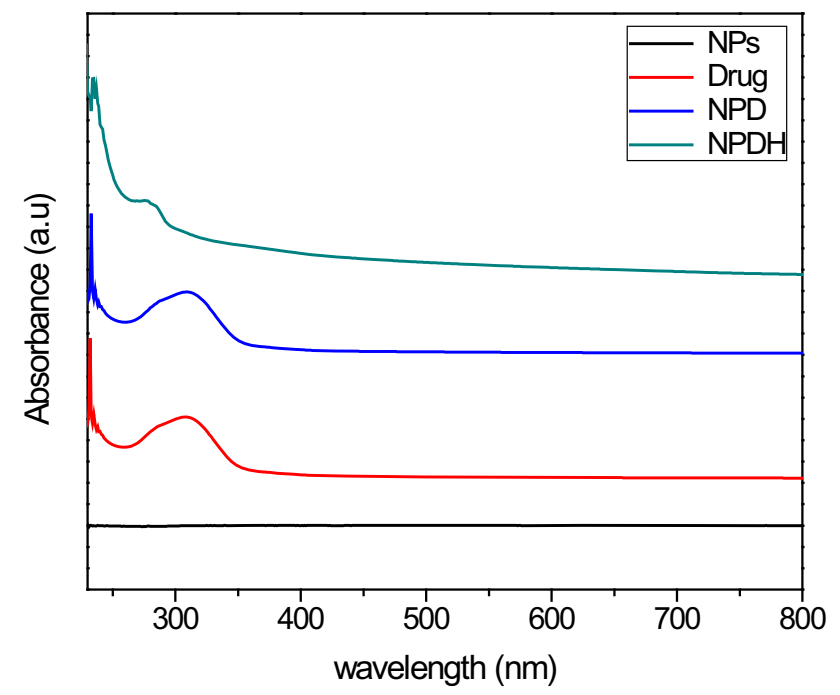

Fig. 6 UV of mesoporous silica nanoparticles after drug loading and gelation

\section{Monastrol release kinetics}

Figure 7 displays the in vitro release studies which are done by simple dialysis method. About $40 \mathrm{mg}$ of monastrol-loaded nanoparticles are transferred to dialysis tubing and suspended in $40 \mathrm{~mL}$ of freshly distilled water at room temperature without any magnetic stirring or shaking for $24 \mathrm{~h}$. The drug release was assessed by sampling the contents $(4 \mathrm{~mL})$ of the outer media and recording their absorbance by UV-spectrophotometer after every half an hour. It was observed that in early hour drug was released steadily with a rate of $8.4 \mathrm{mg} \mathrm{cm}^{-3} \mathrm{~h}^{-1}$, but with the passage of time, drug release reached an equilibrium state

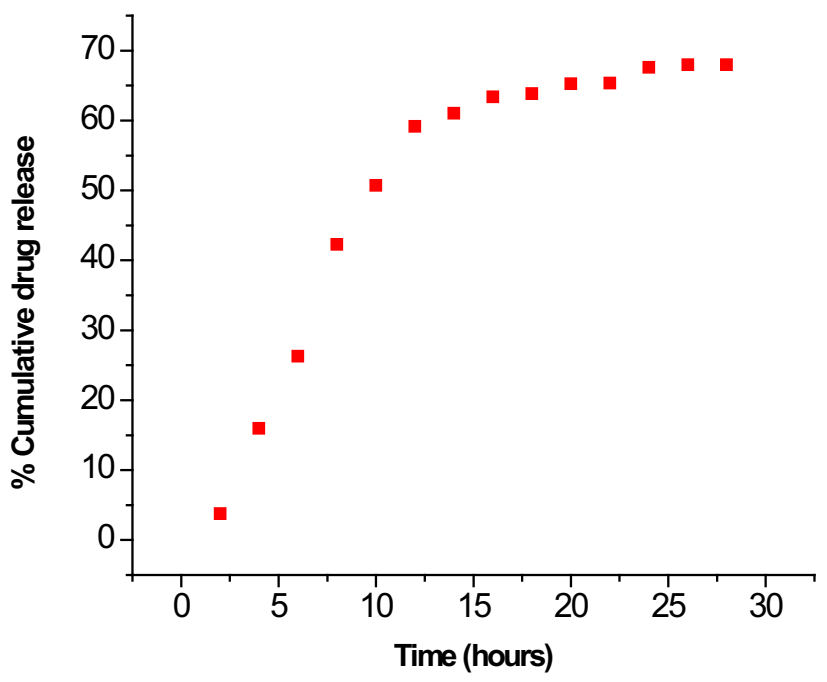

Fig. 7 Kinetics of drug release

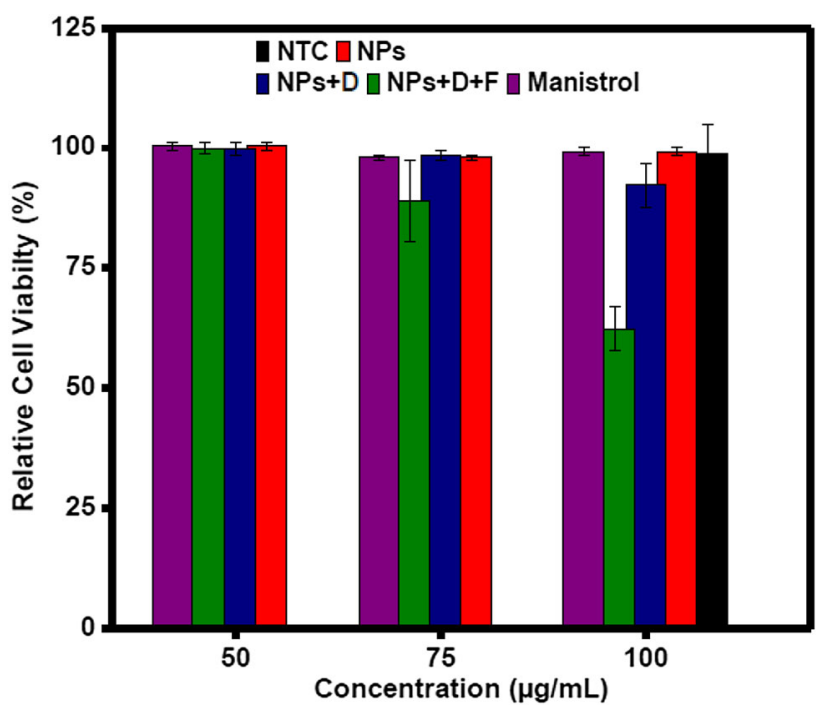

Fig. 8 Relative cell viabilities of NPs and their drug-loaded nanoconjugates. The bars indicate the mean $\pm \operatorname{SD}(n=3)$ 


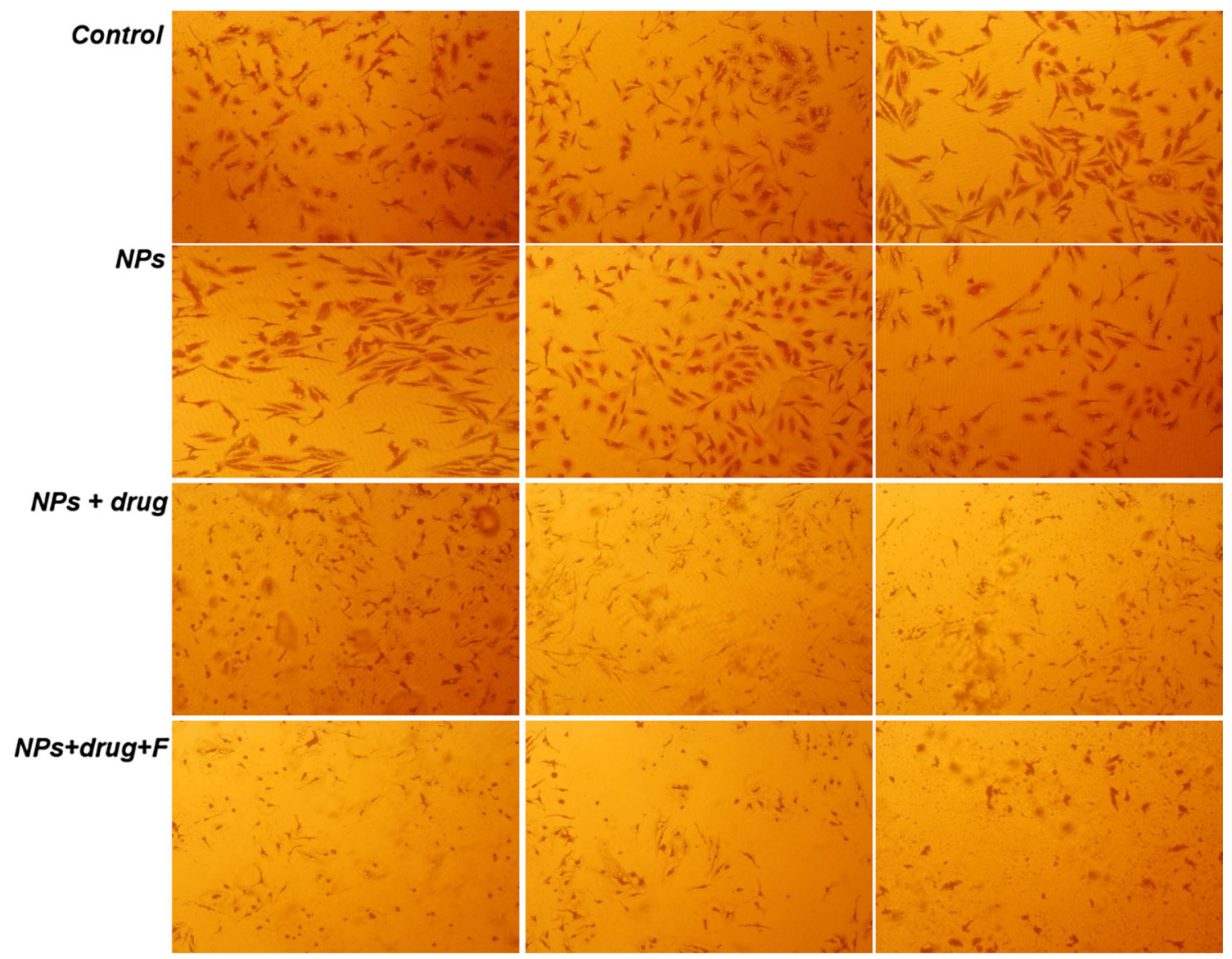

Fig. 9 Phase contrast microscopic images of heLa cells incubated with NPs and their drug conjugates

which shows sustained release behaviour of monastrol with time.

\section{In vitro innate cytotoxicity}

The effect of free drug and drug nano-carrier on inhibition of cell proliferation in HeLa cells was monitored after addition of $0.34,0.26$ and $0.17 \mu \mathrm{M}$ of Monastrol contained in 100, 75 and $50 \mu \mathrm{g} / \mathrm{mL}$ of NP-D and NP-D-F. The effects of Monastrol, SNPs, NP-D and NP-D-F on cell viability were determined using MTT assay. The absorbance measurement, by using this assay, reflects the total metabolic activity of a cell population and is therefore an indirect measurement of cell proliferation. Although commonly used as a viability assay, the MTT assay more specifically represents a measure of mitochondrial function.

The cytotoxicity of the pure NPs, NP-D and NP-D-F was compared with NTC and the pure monastrol. Even at concentrations as high as $100 \mu \mathrm{g} / \mathrm{mL}$, Si NPs had shown no cytotoxicity on the human cervical epithelial malignant carcinoma (HeLa) cell viability (Fig. 8). This demonstrated the high biocompatibility of Si NPs as drug delivery agents. The very low activity of monastrol at $100 \mu \mathrm{g} / \mathrm{mL}$ displayed its low penetration across the cell barrier at this concentration. NP-D demonstrated a slight toxicity at $100 \mu \mathrm{g} / \mathrm{mL}$ while NP-D-F demonstrated a fairly high toxicity at $100 \mu \mathrm{g} / \mathrm{mL}$ indicating that folate conjugation of silica nanoparticles were favourable in the selective uptake of Monastrol across the cell membrane.

Phase contrast microscopic images of HeLa cells incubated with NPs and their drug conjugates is shown in Fig. 9. Our presented system presents a reliable method for selective monastrol release to the diseased cancer cells by in vitro.

\section{Conclusion}

From the above-mentioned study, it can be concluded that the mesoporous silica nanoparticles were successfully synthesized by sol-gel method. These particles were then characterized by BET surface area and pore size distribution analyses, UV, FTIR, XRD and SEM. The surface area of MSNPs was found to be $328 \mathrm{~m}^{2} / \mathrm{g}$ and the maximum pore size of silica nanoparticles was about $5.6 \mathrm{~nm}$. Monastrol drug was loaded on MSNPs for controlled drug 
delivery, where UV and FTIR analyses were done on every important step. The cell viability was observed to decrease with NPD and decreases further after loading folic acid. MSNPs with high payloads of monastrol were effectively delivered into the cancer cells in vitro. Folic acid conjugation ensued the targeted delivery, whereas CMC-based hydrogel of MSNPs effectively packed monastrol deep into the pores of MSNPs.

\section{Compliance with ethical standards}

Conflict of interest The authors declare that they have no conflict of interest.

Ethical standards The manuscript does not contain clinical studies or patient data.

Open Access This article is distributed under the terms of the Creative Commons Attribution 4.0 International License (http:// creativecommons.org/licenses/by/4.0/), which permits unrestricted use, distribution, and reproduction in any medium, provided you give appropriate credit to the original author(s) and the source, provide a link to the Creative Commons license, and indicate if changes were made.

Publisher's Note Springer Nature remains neutral with regard to jurisdictional claims in published maps and institutional affiliations.

\section{References}

Bharti C, Nagaich U, Pal AK, Gulati N (2015) Mesoporous silica nanoparticles in target drug delivery system: a review. Int $\mathbf{J}$ Pharm Investig 5:124-133

Chen Y, Zhang H, Cai X, Ji J et al (2016) Multifunctional mesoporous silica nanocarriers for stimuli-responsive target delivery of anticancer drugs. RSC Adv 6:92073-92091

Fent K, Weisbrod CJ, Wirth-Heller A, Pieles U (2010) Assessment of uptake and toxicity of fluorescent silica nanoparticles in zebrafish (Danio rerio) early life stages. Aquat Toxicol 100:218-228

Ganesh M, Lee SG (2013) Synthesis, characterization and drug release capability of new cost effective mesoporous silica nanoparticle for Ibuprofen drug delivery. Int J Control Autom 5:207-216

Hassan SF, Rashid U, Ansari FL et al (2013) Bioisosteric approach in designing new monastrol derivatives: an investigation on their ADMET prediction using in silico derived parameters. J Mol Graph Model 45:202-210
Jaganathana H, Godin B (2012) Biocompatibility assessment of Sibased nano and micro-particles. Adv Drug Deliv Rev 64:1800-1819

Jong WHD, Borm PJA (2008) Drug delivery and nanoparticles: applications and hazards. Int $\mathbf{J}$ Nanomedicine 3:133-149

Liu T, Li L, Teng X, Huang X, Liu H, Chen D, Ren J, He J, Tang F (2011) Single and repeated dose toxicity of mesoporous hollow silica nanoparticles in intravenously exposed mice. Biomaterials 32:1657-1668

Maliga Z, Kapoor TM, Mitchison TJ (2002) Evidence that monastrol is an allosteric inhibitor of the mitotic kinesin Eg5. Chem Biol 9:989-996

Mohseni M, Gilani K, Mortazavi SA (2015) Preparation and characterization of rifampin loaded mesoporous silica nanoparticles as a potential system for pulmonary drug delivery. Iran J Pharm Res 14:27-34

Nazir S, Hussain T, Ayub A, Rashid U, MacRobert AJ (2014) Nanomaterials in combating cancer: therapeutic applications and developments. Nanomedicine 10:19-34

Prokopcova H, Dallinger D, Uray G, Kaan HY et al (2010) Structureactivity relationships and molecular docking of novel dihydropyrimidine-based mitotic Eg5 inhibitors. Chem Med Chem 5:1760-1769

Rashid U, Batool I, Wadood A, Khan A et al (2013) Structure based virtual screening-driven identification of monastrol as a potent urease inhibitor. J Mol Graph Model 43:47-57

Rosenholm JM, Sahlgren C, Linden M (2011) Multifunctional mesoporous silica nanoparticles for combined therapeutic, diagnostic and targeted action in cancer treatment. Curr Drug Targets 12:1166-1186

Sahoo SK, Parveen S, Panda JJ (2007) The present and future of nanotechnology in human health care. Nanomedicine 3:20-31

Schmidt M, Bastians H (2007) Mitotic drug targets and the development of novel anti-mitotic anticancer drugs. Drug Resist Updat 10:162-181

Suri SS, Fenniri H, Singh B (2007) Nanotechnology-based drug delivery systems. J Occup Med Toxicol 2:16-21

Waseem M, Mustafa S, Naeem A, Koper GJM et al (2010) Physiochemical properties of mixed oxides of iron and silicon. J Non-Cryst Solids 356:2704-2708

Wilczewska AZ, Niemirowicz K, Markiewicz KH, Car H (2012) Nanoparticles as drug delivery systems. Pharm Rep 64:1020-1037

Yu K, Grabinski C, Schrand A, Murdock R, Wang W, Gu B, Schlager J, Hussain S (2009) Toxicity of amorphous silica nanoparticles in mouse keratinocytes. J Nanoparticle Res 11:15-24

Zhang Y, Zhi Z, Jiang T, Zhang J et al (2010) Spherical mesoporous silica nanoparticles for loading and release of the poorly watersoluble drug telmisartan. J Control Release 145:257-263

Zhang Y, Wang J, Bai X, Jiang T et al (2012) Mesoporous silica nanoparticles for increasing the oral bioavailability and permeation of poorly water soluble drugs. Mol Pharm 9:505-513 